\title{
Study on the Maintenance and Management Technology of Computer Software
}

\author{
Jixin Xing \\ Langfang Polytechnic Institute, Langfang, 065000, China
}

Keywords: Computer software, Maintenance and management, Technology

\begin{abstract}
Computer software is an important part of computer, whose running security has a direct impact on the computer. In addition, the maintenance and management technology of computer software plays a key role in software running. Therefore, in order to ensure the smooth running of computer software, in this paper, it studies the maintenance and management technology of computer software, and puts forward strategies to improve the maintenance and management technology of computer software, so that to ensure the software runs more reliably.
\end{abstract}

\section{Introduction}

Computer is mainly divided into two parts: software and hardware. For both, the maintenance and management of software seems more important, because once the computer hardware has any problem, professionals can repair it and ensure the reliable running of the computer. However, if there is the improper maintenance and management of computer software, once there is a failure, it will lead to system failure, so the computer software is very important. Therefore, we must enhance the research intensity of software, as well as the maintenance and management of software, so that to ensure its safe running.

\section{Computer Software Overview}

Computer software refers to the programs and documents in the computer, which are important parts of the computer. Generally speaking, computer software can be divided into two categories: one is the application software and the other is the system software. System software mainly has the function of development and maintenance, controlling the hardware and software resources, and providing convenient operation interface for users to fully use the resources in the system software. System software also includes other subsystems, such as operating system, and database management, etc. Of course, the operating system is the core part of the software, which coordinates the operation of other system, and reasonably allocates and schedules resources to achieve the transferring and sharing of information between users. Currently, in the design of the program, programmers will use programming language software to process data. In short, each subsystem in the system software has its own unique function and role, so that to provide good and convenient conditions for users to use computer software. For application software, it needs to have a powerful database to support its operation, which is mainly to be applied in specific areas to compile the corresponding software product. For example, application software includes e-commerce system and office software, and because the application software is developed with the combination of different areas, so it has more types and broader covering area. Through continuous exploration of human on science and technology, computer software has a broader development space towards intelligent and integrated development.

\section{Problems exist in the Daily Management and Maintenance Technology of Computer Software}

\section{Lack of Computer Software Technology}

If the computer software wants to be operated safely and reliably, it must have good software maintenance and management technology; however, the lack of computer software maintenance and management technology at present affects the user of computer software for users. With the 
continuous advance of science and technology, human beings have entered in the network age, so the network is closely related to computer software. Due to the security of network is not high, and the paste software programs are lack of bug fixing abilities, so when there is an unsafe factor in the network, the ability for the system to resist and kill the virus is low, the system repair is very slow, and even the system will breakdown. In short, due to the software programming system is lack of self-repair capability and the software maintenance and management technology, which affects the normal experience of software for users.

\section{Threats of Internet towards Software}

Due to the Internet combines with the computer software, so the difficulty of maintenance and management of computer software increases. At present, there are greater risks exist on the network and many users are lack of network knowledge, so when users operate the software interface, it is easy to appear the non-standard operation phenomena. In the process of using computer software, due to the improper operation and users and the lower network security, the Internet threats the software and damages the computer software system ${ }^{[1]}$. When a problem occurs in the Internet, users are lack of maintenance and management capacity and the lack of computer software maintenance and management technology, finally leading to many obstacles for the computer software system.

\section{Non-standard Use of Software for Users}

Computer software is the product developed by human beings, so in the process of software development, it needs to set the corresponding functions for users or software developers to maintain and manage the software regularly. In the operation of software process, due to users are lack of software basic knowledge and do not pay attention to routine maintenance and management of software, even the improper use of software, resulting in the damage of computer software system. When operating the software, some users fail to operate in accordance with standard procedures, but click the function module randomly, or due to the slow running of system and forced close of software program, part of the software lose the data due to the weakness of self-repair ability, giving users some unnecessary troubles. Meanwhile, some users do not pay attention to the management of the software, do not upgrade the software timely, or do not look for professionals to repair the software system, and do not operate normatively, thereby affecting the normal operation of the computer software.

\section{Imperfection of Software Backup Function}

Computer software is researched and designed with the combination of different fields, so it covers a relatively wide range. Through the software, users process data or other content, and every element involved is very important, and users need to save the data in a long term and then use them. Therefore, the backup function of the computer software is very important; however, some software are lack of data backup function, even it requires users to manually back up data. Therefore, the data are not easy to lose, and its security reduces and it also increases the workload of the user. Of course, even some software itself has a backup function, due to the program design is unreasonable, backup technology is imperfect, improper operation of users or a sudden power failure, it will lead to partial loss of date, or even data irreparability, so that to seriously influence the normal use of software for users.

\section{Strategies to Improve the Maintenance and Management technology of Computer Software}

\section{Improve the Maintenance and Monitoring System of Computer Software}

To improve the maintenance and management technology of computer software, we must improve its maintenance and monitoring system. Firstly, it adopts user access and control program. Due to this system determines whether the user has the permission to use this computer, it will authenticate the identity of user, so that to strengthen the management and control of computer software. Secondly, it uses software encryption technology, and this technology can be considered from the password setting and user identity in the information of software. Encryption technology can effectively 
prevent hackers from stealing personal information, avoid missing information, enhance the security of user information, reduce the destroy of software function and information, thereby enhancing the maintenance of computer software. Then, it uses anti-virus firewall technology to improve network security and effectively manage the computer software. Anti-virus firewall technology enables the computer to monitor the gateway of computer and Internet, virus and the information influencing the computer, so that to protect the safe operation of computer software. Finally, it embeds bug protection scanning technology. If users do not operate the computer software regularly, the software system will be damaged, as well as appears management bug, so this technology can repair the bug and restore the integrity of the software system ${ }^{[2]}$. In short, to improve the maintenance and management technology of computer software, we must integrate these technologies into the maintenance and management of computer software, so that to maintain and manage the computer software very well.

\section{Strengthen Maintenance and Management Intensity of Computer Software}

Maintenance and management are important factors to ensure reliable operation of software, so in order to facilitate the continuous operation of computer software and to reduce the frequency of failure occurrence, it must regularly remove trashes in the system and clear the caches, therefore, it will clean up more spaces for the system. Users need to timely repair the system, upgrade patches, diagnose the failures appear in the system, and ask professionals to deal with system problems. When operating the software, users needs to follow norms and avoid system crash due to poor operations. In addition, users compute the software backup, regularly check the operation of software, check the security of network, and repair the bugs in the system timely. By strengthening the maintenance and management of computer software, it improves the operating efficiency of computer software.

\section{Improve Terminal Technology}

Improving terminal technology can enhance the maintenance and management of computer software and ensure the reliable operation of software Fore improving the terminal technology of software, it can be considered from the following two aspects: firstly, diskless workstation setup. When the computer is running in a network environment, because it will go through the server in the starting process, so the diskless network also means the diskless workstation. Due to the diskless network environment can reduce costs, so the fund used to purchase the disk can be used to improve other technologies. In addition, when the computer software has some failures, the diskless workstation is more conducive for technicians to maintain. If there is a disk, the sudden power cutoff will result in data loss, while the diskless workstation do not have such situation, and it has a lager memory, which makes the operation efficiency of computer motherboard improve significantly. Meanwhile, the diskless network environment can reduce the threats to the system configuration when the system is under attack. Secondly, diskless terminal network creation. It is a new terminal technology, and system components exist in the terminal without maintenance, so it can be automatically upgraded. In addition, it can contact with the server through TCP/IP and remote way, and contact with the server via LAN, while the diskless terminal applies to multiple computers without affecting the work of each computer ${ }^{[3]}$. In addition, the diskless terminal does not need to back up, so only the regular upgrade can ensure the functional completeness of software system and the smooth operating of the system.

\section{Improve the Restoration Technology of Software}

For improving the maintenance and management technology of computer software, the software function must be improved, so the software backup function has a very important function, and plays a key role in improving data security. In order to improve the quality of software backup function, it needs to perfect the restoration technology of computer software. Firstly, using Ghost to back up. Ghost backup is the best way for data restoration, and Ghost backup does not run through the entire process of using the software, but it is selective. When the computer software system is installed completely, other basic software are also installed to carry out backup processing. It should be noted that, if the user's computer is brand new, it needs to divide the disk, then to back up after operating the computer, and finally to divide the disk. In short, the division of the disk does not have a unified 
standard, but according to the needs of users. After completing the disk division, the computer can be operated normally. Of course, the backup content should not be placed in Disk C, but can be placed in other disk. While the format of other disk is FAT32, so in order to improve the security of data, users can save the data in the CD. With the development of science of technology, Ghost one-key backup also can achieve the desired effect, and its operation is more convenient. Secondly, the installation of disk protection card is also the basic requirement to perfect the restoration technology ${ }^{[4]}$. Disk protection card and MBR are cooperated with each other. Through managing BIOS and interrupting the work of INT13, it will save the information such as FAT and interrupt vector in the recovery card, or save the information in the disk. In this case, the interrupt vector in the recovery card replaces the interrupt vector table, and saves the information in the recovery card to achieve the virtual restoration. Finally, a good restoration technology also has a Ghost maintenance tool. For the places with large computer using frequency such as schools and large enterprises, due to the large amount of information, so it must have the system maintenance conditions. Ghost Corporate Edition has a powerful function, which enables the maintenance and management of software information. Ghost Corporate Edition is installed on a Windows system, the first step is to install the sever port, and the second step is to install the client. And it needs to take the driving factor into account, then it can be used normally after completing installation ${ }^{[5]}$.

\section{Maintenance and Management Technology Outlook of Computer Software}

Improving the maintenance and management of computer software can enhance the running ability of software, and improving the restoration of software and terminal can provide a favorable basis for the reliable operation of software. Through continuously innovating technologies, our computer software researchers make the functions of the computer software system more powerful. Of course, science and technology are endless, so the development, maintenance and management technology of computer software also need to be further improved. In order to promote better development of computer software, and provide customers with good service platforms, we must have a outlook on the maintenance and management technology to make the computer software towards intelligentization and integration. Especially for the further improvement of restoration technology, the future software backup does not require Ghost mirroring and maintenance to process, only requires one-key reset to complete backup, and it not only does not require complex and professional enterprise maintenance tool to install, but also has a stronger system backup capability and greater security. In addition, it needs to upgrade the software repair capacity, and strengthen the research of anti-virus technology, so that to make the security of computer software system greater and to improve the operation efficiency.

\section{Conclusion}

In the progress of science and technology, computer software technology is more mature, so in order to provide users with a good software platform, it needs to improve the maintenance and management technology of computer software, and promote the smooth operation of software. Of course, the maintenance and management technology of computer software can not remain at the current level, so human beings need to improve the technology to increase the reliability of computer software.

\section{References}

[1] He Yunyong. Discussion on the Maintenance and Management Technology of Computer Software. Practical Electronics, 2013(15):78-78.

[2] Zeng Zhiyong. Exploration on the Computer Maintenance and Management Technology. Technology and Market, 2014(2):34-35. 
[3] Bao Yueling. Software Information Maintenance and Management under Computer Software Platform. Information Technology \& Informatization, 2014(4):190-192.

[4] Han Zongqiu. Analysis on System Maintenance and Management of Computer Software. Orient Culture Week, 2014(13):113-113.

[5] Wang Liping. Exploration on Maintenance and Management of Computer Software System. Computer CD Software and Application, 2012(10):66-67. 